\title{
THE ANTINEOPLASTIC ROLE OF STAT5 INHIBITION IN BCRABL1- POSITIVE CELLS EXPOSED TO PIMOZIDE ALONE AND IN COMBINATION WITH DASATINIB AND PONATINIB
}

Doctoral Dissertation of:

Dr. Marco Santoro

Supervisors:

Prof. Francesco Passamonti

Prof. Francesco Di Raimondo

Tutor:

Prof. Sergio Siragusa

The Chair of the Doctoral Program:

Prof. Antonio Russo 


\section{INDEX}

1. Abstract

Pag 3

2. Summary

Pag 6

3. CHAPTER 1 Background Rationale and Objectives

Pag 9

4. CHAPTER 2 Materials and Methods

Pag 21

5. CHAPTER 3 Results

Pag 23

6. CHAPTER 4 Discussion

Pag 24

7. CHAPTER 5 Tables and Figures

Pag 27

8. Bibliography

Pag 30 


\section{Abstract}

EVEN though the last decades have seen the success of the targeted treatments for BCRABL1-positive Chronic Myeloid Leukemia (CML), with the outstanding achievement of placing selected patients into the so called treatment-free remission, a minor part of these subjects face TKI resistance and/or intolerance. Resistance is a challenging point in the clinical management of CML, occurring in approximately $10-20 \%$ of $\mathrm{CML}$ cases, due to several mechanisms, among which point mutations of the BCR-ABL kinase domain, BCRABL overexpression or alternative splicing, sub-efficient plasma concentration of the inhibitor and abnormal drug efflux/influx.

The Signal Transducer and Activators of Transcription (STAT) proteins are a family of transcription factors commonly involved in multiple intracellular tasks and pathways, among which survival and proliferation. The STAT family is made up of seven factors, each one with a specific role. In particular, STAT3 and STAT5 (A and B) are strictly involved in cell survival and proliferation and thus are commonly implied in the pathogenesis of many neoplasms. STAT5 is of peculiar interest for its critical role in cellular differentiation, adipogenesis, oncogenesis, immune function and is known to be 
constitutively activated after the BCRABL1 effect in CML cells. STAT5 expression has been strictly linked to BCRABL1 mutations and disease progression to accelerated and blast phase and may thus represent a significant target to overcome resistance to TKI in CML.

In 2011, Nelson et al. evidenced the effect of STAT5 inhibition exerted by a Pimozide, a commonly used neuroleptic drug. As far as we know, literature reports experiments involving STAT5 inhibitors in association with BCRABL inhibition only with first generation inhibitor imatinib. There is no news about the association between STAT5 inhibitors and newer generations of TKIs.

The aim of this study is to explore the antineoplastic role of the STAT5 inhibitor Pimozide in association with $2^{\text {nd }}$ and $3^{\text {rd }}$ generation TKIs, dasatinib and ponatinib respectively, and to identify the cytotoxic in vitro concentrations.

For the purpose of the study, K562 cell line was used to simulate the frame of a classical Chronic Myeloid Leukemia disease. The cytotoxic effect was evaluated by the Trypan blue dye exclusion test. K562 cell lines were exposed to pimozide alone and in association with ponatinib and dasatinib at different concentrations to explore the drugs association effect and the in vitro cytotoxic concentrations.

Pimozide showed a synergic effect when associated with ponatinib and dasatinib in survival inhibition of K562 cell lines. This results are of note and pave the way for a possible in vivo associations. Moreover, an extension of the present study is evaluating the cytotoxic effect on CML leukemic stem cells (LSC), aiming at revealing whether STAT5 inhibition may determine a more profound 
effect on CML disease and may overcome the LSC escape mechanisms on which TKIs are not active. 


\section{Summary}

Chronic Myeloid Leukemia $(\mathrm{CML})$ is a clonal myeloproliferative disease, originating by the occurrence of a translocation between chromosomes 9 and 22 in a hematopoietic stem cell, that generates the chimeric gene BCR$A B L$. The BCRABL chimeric protein works as an altered tyrosine kinase, constitutively triggering the proliferation signal in the clone, and thus determining a finally differentiated hyper-proliferative phenotype in the affected subject.

In 2001, the knowledge of this main pathogenic mechanism lead to the synthesis of the so called "magic bullet" Imatinib, a small molecule tyrosine kinase inhibitor, purposely designed for blocking the BCR-ABL adenosyntryphosphate (ATP) kinase domain and consequently interfering with the pathogenesis of CML itself (1). For decades, the tyrosine kinase inhibitor (TKI) imatinib and its more potent derivatives dasatinib, nilotinib, ponatinib and bosutinib (2) have been used to treat CML with high rates of optimal responses and achieving previously prohibited goals, like maintaining offtherapy remissions in selected cases (3-5). The use of BCRABL-inhibiting TKIs have completely changed the CML prognosis, critically moving the survival curve of CML straight next to the one of the general population and drawing a wide fork respecting to the one of CML patients treated with other previous treatment options, such as interferon (IFN), hydroxycarbamide 
(HU) or hematopoietic stem cells transplantation (HSCT) (6-8). However, TKIs can be discontinued with benefit only in a slight minority of patients, while in the largest part of CML cases TKIs have to be assumed lifelong. Eligibility criteria for drug discontinuation during CML remission have been evaluated and recommended by the Gruppo Italiano per le Malattie EMatologiche dell'Adulto (GIMEMA) in 2019, taking into consideration different variables: disease risk (calculated with the EUTOS long term survival score), patient's features, presence/absence of the need for TFR, first line TKI (4).

Previous studies have documented the presence of BCRABL1 positive leukemic stem cells in the bone marrow of TKI-treated patients achieving deep molecular response (DMR). These primitive CML cells are capable of growth in colonies, even if BCRABL is not detected by polymerase chain reaction in those patients. Patients with no detectable LSC in the bone marrow can subsequently relapse during the treatment-free remission follow-up, and this is consistent with the idea that LSCs sometimes cannot be isolated because of technical limitations. Other studies revealed persisting LSCs decrease their BCRABL expression in patients in deep molecular response, respecting to the time point of diagnosis (9-13).

Other experiments have revealed that resistance to TKIs can be overcome when acting on another molecular target, represented by the STAT proteins. Pimozide, a drug already used for its neuroleptic functions, and some of its derivatives, have been tested in preclinical models and may inhibit the growth of K562 colonies (a cell line expressing BCRABL).

We here present the preliminary results of a preclinical study aimed at demonstrating the effect of growth inhibition of a CML cell line (K562 cells) 
when exposed to pimozide with and without second and third generation TKI, respectively dasatinib and ponatinib. 


\section{Background, Rationale and Objectives}

\subsection{Chronic Myeloid Leukemia}

According to the Surveillance, Epidemiologic and End Results Programme (SEER) CML represents $0.5 \%$ of all new cancer cases in the US, with a number of estimated new cases in 2021 of 9,110 (14). Based on age-adjusted data of the 2014-2018 period, the rate of new CML cases in the US is 1.9 per 100,000 persons per year (14). A recent study conducted from the Swedish registry on a total of 2,662 patients with CML diagnosed between 1973 and 2013, aged 50 years or older, revealed that life expectancy of CML patients increased by the year of diagnosis and is higher for younger patients. The median age at diagnosis of CML was 69 years (6).

A study published on Lancet in 2015, retrieved data from six consecutive or parallel prospective clinical trials on TKI-treated CML patients at a single institution from July 2000 to September 2012. The analysis revealed a 5-year survival comparable to that of the general population, with a median follow-up of 99.4 months (interquartile range 44.9-121.6) (16). 
Chronic Myeloid Leukemia (CML) is a neoplasm arising from the myeloid-committed hematopoietic stem cell, characterized by the translocation between the chromosome 9 and $22(\mathrm{t} 9 ; 22)$, that determines the formation of a chimeric gene, BCRABL, named after the two genes that are involved in the chromosomal aberration. ABL (Abelson) is a tyrosine kinase with the physiological role of prevent apoptosis and stimulate proliferation. In the chimeric molecule $\mathrm{BCRABL}$, the kinase is constitutively active and determines the hyperstimulation of downstream pathways leading to cell survival and proliferation.

The classical presentation of CML is made up by: leukocytosis usually with White Cell Count $>30 \times 109 /$ liter with normally matured neutrophils, anemia (or normal hemoglobin values), different degree of thrombocytosis, splenomegaly from slight to severe, systemic symptoms (fever, swelling, unexpected weight loss).

CML may be diagnosed in different disease phases: Chronic Phase (CP), Accelerated Phase (AP) and Blast Crisis (BC). The most of diagnosis are made in the $\mathrm{CP}$, that assures less degree of clinical manifestation and, in fact, it is usually an occasional diagnosis during control exams, in the absence of a specific clinical manifestation.

Diagnosis is made according to the WHO 2016 revision of criteria for the diagnosis of myeloid malignancies, that is specific morphological findings at the peripheral blood smear and the presence of the chimeric BCRABL1 mRNA transcript revealed with the Polymerase Chain Reaction (PCR) on the peripheral blood too. Bone marrow study is not mandatory, but sometimes it is useful to obtain adequate material for baseline conventional cytogenetic study. This last exam is 
recommended at the moment of diagnosis, before starting TKI to search for the so called Adjunctive Cytogenetic Alterations (ACA), chromosomal abnormalities other than $\mathrm{t}(9 ; 22)$ that account, if present, for a worse prognosis and make diagnosis of Accelerated PhaseChronic Myeloid Leukemia (AP-CML).

The 2016 WHO revision updated the criteria or the diagnosis of AP$\mathrm{CML}$, defining it as the presence of at least one of the following: persistent leucocytosis unresponsive to treatment; persistent thrombocytosis unresponsive to treatment; thrombocytopenia; splenomegaly not responsive to treatment; basophils at least $20 \%$ on peripheral blood; blasts $10-19 \%$ on peripheral blood or bone marrow; ACA at the diagnosis or in every moment of the follow up. AP-CML is at high risk of transformation into a blast-phase (BP), that is an acute leukemia evolution, with myeloid or lymphoid features, and is frequently less prone to molecular response with first generation TKI imatinib, while is indicated upfront dasatinib high-dose $(140 \mathrm{mg}$ a day).

When none of these criteria is present and the blasts count in peripheral blood or in bone marrow is less than $10 \%$, chronic phase chronic myeloid leukemia (CP-CML) is diagnosed (17).

\section{Principles of TKI therapy}

BCRABL1 tyrosine kinase inhibitors were designed properly to contrast the constitutive activation of the chimeric protein and thus block the survival and proliferation signal that is the key pathogenic moment of the disease. Therefore, TKIs are small molecules built up to switch off the CML pathogenesis. 
The targeted site is the kinase domain, and in particular the ATP binding site, that has the function of linking an ATP molecule on the TK, from which energy and a phosphate are derived to fulfil its enzymatic property. The TK active sites may take two different conformations: the active and the inactive status. In the active status, the so called activation loop (A-loop), controlling the access to the catalytic site, is "open" and the control motif (an aspartatephenylalanine-glycine conserved sequence - DFG motif) is placed in the inward position and may operate the ATP stabilization in order to place the ATP molecule in the right position to operate the dephosphorylation. On the opposite, the inactive status consists of a "closed" A-loop and an inward DFG position, thus preventing the ATP from being available to the enzyme for dephosphorylation.

TKIs are accordingly classified as type I inhibitors when they compete directly with the ATP binding, recognizing and blocking the active conformation of BCRABL, and type II inhibitors, when they recognize and stabilize the inactive conformation and only secondarily prevent the ATP from finding the path to the dedicated binding site (19). Of the available TKIs, only dasatinib acts as a pure type I inhibitor, while imatinib, nilotinib and ponatinib operate a type II inhibition and bosutinib acts both as a type I and II inhibitor. Type II inhibitors have more stringent binding rules and thus are more prone to suffer from resistance, when a mutation occurs to the binding site, but express a more specific inhibition on the ABL molecule; on the other hand, type I inhibitors suffer less from possible mutational-based resistance, but may recognise also non- $B C R A B L$ targets and consequently carry the risk for adverse events coming from other TK inhibition (i.e. SRC kinases inhibition may determine serosa effusions) (20). 
Since 2001, when the first generation TKI imatinib become available for clinical practice the management of the disease has dramatically changed, with an impact on prognosis to the point of obtaining overall survival comparable to the general population. Nilotinib, dasatinib, bosutinib and ponatinib were then developed and commercialized, with the evidence of being faster and even more effective than imatinib at reaching deep molecular responses $(21,22)$.

These striking results obtained by 2 nd and 3 rd generation TKIs, however, usually came along with a heavier toxicity profile. Taking into consideration the excellent rate of survival that we showed in the Epidemiology section, a relevant aim of the long term treatment is to optimize the quality of life of a person who assumes a TKI.

Treatment-free remission has recently been considered safe and convenient for a subset of patients with CML who fulfil some criteria, among which a deep molecular response and a long period of TK inhibition with stable molecular response. Since the first time that TFR was taken into consideration, in the STIM1 study, it was successfully and safely obtained in CML patients treated with imatinib or second generation TKIs (23-26). Reached TFR percentages vary between studies and ranged between 38 and $70 \%$, but in all of them safety was similarly acceptable and consistent. Periodic molecular monitoring permit to achieve an eventual early diagnosis of loss of MMR in patients which fail the TFR. More than $90 \%$ of the patients who lost the MMR and restarted the previously suspended TKI reached the

Therefore, selected patients are nowadays considered for TKI suspension, considered to be a safe procedure, at least for centres 
that have access to robust sensitive molecular monitoring, assuring a short-term reporting of the molecular response (27).

A step forward has been recently done with the development of a new $A B L$ inhibitor, asciminib, that links to the myristoylic site of the molecule, outside the ATP cleft. That site has the role of an allosteric regulator and asciminib is capable of determine a significant modification to the catalytic conformation of the $A B L$, thus preventing its constitutive activity. Data on large cohorts for safety and efficacy of asciminib are still lacking and the drug is nowadays available in a managed access program for patients without any other possible treatment. Asciminib was active in patients with CML who had withdrawn TKIs for documented resistance or unacceptable toxicity, including patients with ponatinib-failure and the ones with the T315I mutation (28). Limited evidence is available for the use of omacetaxine, a molecule that operates as an inhibitor of protein translation, blocking the synthesis of proteins among which BCRABL1 (29). Donor transplant of Hemopoietic Stem Cells is still an option that has to be very specifically selected and chosen, when no other alternatives are available (30).

BCRABL-dependent and -independent Leukemic Stem Cells escape paths

Leukemic Stem Cells (LSCs) persistence appears to be a frequent (if not systematic) event occurring in patients with CML obtaining any depth of response to TKI treatment. CML LSCs can be defined as primitive stem cells that express BCRABL 1 , have a higher capacity of engraftment than bulk CD34+ cells, are prone to genomic instability 
and have impaired DNA-damage. The advantage in proliferation provided by the presence of the chimeric protein BCRABL1 and the concomitant capacity of laying into a quiescent status seems to be a paradox, and though it is the most likely means by which the CML LSCs may provide an escape form the TKI inhibition and thus persist. This was evidenced by both in vitro and in vivo trials concluding that CML LSCs were not "oncogene-addicted" and that targeting of BCRABL1 kinase activity alone would not eliminate them $(31,32)$.

Taking into consideration the optimal responders, the mechanism of alternative splicing of the BCRABL1 transcript is frequently a cause for failure to achieve DMR and thus to be considered for TFR (33). A recent article by Kinstrie et al. reports how quiescent LSC express CD93 at a high intensity in flow cytometry and persist in a population of CML patient samples who demonstrate molecular relapse on TKI withdrawal (34). In an article published in 2020, Jeanpierre et al. demonstrated that a sub-fraction of CML LSC relies on the bone morphogenic protein-4 (BMP4) pathway for quiescence and BCRABL1-independence survival and expresses BMPR1B (BMP receptor 1b). This subset of cells are TKI-insensitive and thus may represent a possible target of drugs aiming to CML LSC-cleavage/killing (35).

BCRABL1 was demonstrated to activate the phosphatidylinositol-3'kinase (PI3K)/AKT signalling, determining, among other effects, FOXO transcription factors phosphorylation and their cytoplasmic localization. FOXO proteins (i.e. FOXO1 and FOXO3a) in the nucleus activate a group of genes capable to direct the cell into its cycle. BCRABL1 constitutive signalling keeps FOXO in the cytoplasm and causes G1 phase arrest and quiescence. Among the FOXO3a 
targets, BCL6 plays an important role in antagonizing p53 and ARF oncosuppressors (37-40).

The Hedgehog $(\mathrm{HH})$ signalling appears to be of interest as a possible BCRABL1-indipendent mechanism of CML LSC immortalization. $\mathrm{HH}$ can bind to Patched and activate a molecular intermediary called Smoothened (SMO) mediator. SMO upregulates transcription factors that lead to the increased MDM2-mediated p53 cleavage and thus determines cell-cycle arrest, resistance to pro-apoptotic signals and LSC quiescence $(41,42)$. In an in vitro model, molecules blocking the $\mathrm{HH}$ pathway have obtained LSC damage in terms of survival and selfrenewal capacity either alone or in combination with $2^{\text {nd }}$ generation TKI Nilotinib (43).

Other molecules are involved in LSC escape mechanisms in presence of a TKI, but we will not focus on this theme anymore, since it's not the aim of the present study.

\subsection{The STAT pathway in hematologic malignancies}

\section{The STAT way}

The major target of the ABL kinase is the Signal Transducer and Activation of Transcription (STAT) proteins family, that is made up by 7 different intracellular proteins that can recognize the activated autophosphorylated kinase with their $\mathrm{SH} 2$ domain and make themselves susceptible to phosphorylation in consequence (44).

When phosphorylated, STATs are disposed to create homodimers with other phospho-STATs and, in the dimer conformation, access the nucleus and link to promoters of specific genes that are related to the 
STAT protein desired effect. STATs are ubiquitary represented in cells that are not terminally differentiated or that are inducible for any kind of reason (virtually all cells). Regardless which kinase directly activates a STAT protein, the cytokine receptor that is linked to that kinase is very selective to the external signal molecule.

\section{STATs in hematological malignancies}

Of primary interest in the pathogenesis of proliferative hematologic diseases, STAT3, STAT5A and STAT5B are assigned to activate genes related with survival, prevention of apoptosis and proliferation. Genes codifying for these three STATs are clustered con chromosome 17 (45). The hematologic compartment widely expresses STAT5A and B in association with different cytokine receptors, among which Stem Cell Factor receptor (SCFR), Granulocyte-Monocyte Colony Stimulating Factor receptor (GMCSFR), G-CSFR, Erythropoietin receptor (EPOR) and Thrombopoetin receptor (TPOR).

Some studies already explored the role of STAT3 and STAT5 in the determination of different hematological malignancies, among which BCRABL1 positive CML, Acute Myeloid Leukemia and JAK2V617Fpositive myeloproliferative neoplasms. There is evidence that some Tcell malignancies also bear STAT3 or STAT5 mutations, with subsequent overexpression of BCL-XL, supporting the cell immortalization, and Cyclin D1, causing proliferation and tumor growth (46-48).

STAT5 was demonstrated to have a major role in the pathogenesis of CML, since BCRABL directly and constitutively activate STAT5 by 
phosphorylation with the effect of promoting cell survival and proliferation. Moreover, STAT5 suppression in CML cells determines apoptosis, increases sensitivity to BCRABL inhibitors even in imatinibresistant CML cells (49). This is of note, when considering the possibility of blocking STAT5 with small molecule inhibitors with a therapeutic purpose in CML patients.

In Acute Myeloid Leukemia (AML) and Acute Lymphoblastic Leukemia (ALL), the chimeric protein BCRABL may be found (especially in a frame of multiple and complex mutations and/or chromosome abnormalities), even if in a minority of the cases. More frequently, in AML mutations affecting the FMS-like tyrosine kinase 3 (FLT3) receptor can be found, conferring constitutive tyrosine kinase activity to the leukemic cells, with a permanent downstream signaling involving pathways among which PI3K-AKT, RAS-MAPK and STAT5 (50-52). A lot of attempts have been made to target the FLT3 signaling in AML with a discreet efficacy but limited durations of responses, mainly because of the developing of escape mechanisms and acquired resistance. The direct targeting of STAT5, in which so many pathways merge together, appears a convenient and possibly effective strategy to strongly inhibit cancer survival and growth.

Chronic Myeloproliferative Neoplasms (MPNs) other than CML frequently express the JAK2V617F mutation or other mutations occurring on genes, such as CALR or MPL, belonging the same set of molecules involved in transduction of the survival and proliferation information from outside the cell to the cytoplasm. V617F mutated JAK2 is a constitutively active tyrosine kinase, conferring cytokine independence to myeloid precursors. In this process of gaining cytokine-independence, STAT5 is absolutely required for 
transformation and induction of a MPN. Experiments on mice with an induced MPN, demonstrated how knocking down the STAT5 signal determines reduction of splenomegaly and normalization of complete blood count cells and abrogates the capability of EPO-independent colony formation of the erythroid precursors. Switching back the STAT5 on restored all the defects obtained with the knock down $(53,54)$. These observations make the MPNs another pathological setting in which a STAT inhibitor may obtain clinical efficacy and success. The three named pathways all of which flow into STAT5 are represented in Figure1.

\section{STAT inhibitors: pimozide and its derivatives}

Pimozide is a drug commonly used for its neuroleptic effect, that also bears a marked capacity of inhibiting STAT5, discovered in 2011 by Nelson and colleagues. The STAT5 suppressing activity is obtained via decreasing its phosphorylation and consequently reducing the expression of STAT5 target genes. When K562 cells are exposed to pimozide, they arrest in the cell cycle and become prone to apoptosis. Among the known side effects, the extrapyramidal syndrome can be a common event.

In 2019, Tolomeo M et al. managed to synthetize pimozide derivatives that ideally do not bear the neurological toxicity of the parent drug, while may achieve a more potent and selective activity (55). The authors preserved the benzimidazolinone-piperidine group, as it resulted to be frequently seen in other active compounds and represents a key component to exert some biological activities. Between the numerous compounds synthetized, 2 in particular were 
significantly more potent than pimozide (derivative 8 and 9). To diminish the risk of developing a central nervous system adverse event (such as the extrapyramidal syndrome reported in the pimozide common use), the authors modified an amine into an amide functional group, but the demonstration of success in the strategy was impossible in the preclinical setting.

\section{Objectives}

As far as we know, literature reports experiments involving STAT5 inhibitors in association with BCRABL inhibition only with first generation inhibitor imatinib. There is no news about the association between STAT5 inhibitors and newer generations of TKIs.

The aim of this study is to explore the antineoplastic role of the STAT5 inhibitor Pimozide in association with $2^{\text {nd }}$ and $3^{\text {rd }}$ generation TKIs, dasatinib and ponatinib respectively, and to identify the cytotoxic in vitro concentrations. 


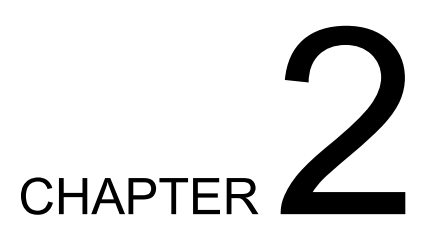

\section{Materials and Methods}

\section{Cell lines and cultures}

For the purpose of the study, K562 cell line was used to simulate the frame of a classical Chronic Myeloid Leukemia disease. K562 express the chimeric oncogene BCR-ABL and is sensitive to its inhibition. Cells were cultured in RPMI 1640 (Gibco Grand Island, New York, USA) containing $10 \%$ foetal calf serum (Gibco), penicillin at a concentration of $100 \mathrm{U} / \mathrm{ml}$ and streptomycin $100 \mu \mathrm{g} / \mathrm{ml}$ (Gibco) and Lglutamine $2 \mathrm{mmol} / \mathrm{lt}$ (Sigma Chem. Co., St Louis, Missouri, USA) in a $5 \%$ carbonic anhydrite atmosphere, at a temperature of $37^{\circ} \mathrm{C}$.

\section{Cytotoxic assays and drug association effects}

The cytotoxic effect was evaluated by the Trypan blue dye exclusion test. To obtain data on the concentrations at which the single drugs determine K562 growth inhibition, 2x105 cells were plated on 25mmdiameter wells with $1 \mathrm{ml}$ of complete medium and different drug concentrations were added as follows: for dasatinib $0.01,0.001$, $0.0005,0.0001,0.00005 \mu \mathrm{mol} / \mathrm{lt}$; for ponatinib $0.01,0.005,0.007$ $\mu \mathrm{mol} / \mathrm{lt}$; for pimozide 10 and $5 \mu \mathrm{mol} / \mathrm{lt}$. In the pimozide-dasatinib 
association experiment, pimozide concentration chosen was 2.5 $\mu \mathrm{mol} / \mathrm{lt}$ and dasatinib 0.00005 and $0.00001 \mu \mathrm{mol} / \mathrm{lt}$. Testing the pimozide-ponatinib association, pimozide concentration chosen was $2.5 \mu \mathrm{mol} / \mathrm{lt}$ and dasatinib 0.005 and $0.0025 \mu \mathrm{mol} / \mathrm{lt}$. Concentrations were chosen according to previous experiences and known preclinical and clinical data on the pharmacodynamics of the inhibiting molecules.

After 48 hours of incubation with the drugs, the percentage of vital cells was determined and expressed in confront to the control noninhibited proliferating K562 cells.

To study the association between drugs, the Webb's fractional product method was applied, calculating the predicted values of inhibition with the equation $c=a \times b / 100$, where $c$ is the predicted value and $a$ and $b$ are the known percentages of growth inhibition obtained with the single drugs. The predicted value of growth inhibition is then compared with the actual value to obtain a ratio that is equal to 1 in a perfect additional effect, less than 1 when synergism exists and more than 1 if the two drugs determine antagonism. 


\section{Results}

K562 cell cultures with dasatinib, ponatinib and pimozide, each one tested alone revealed after 48 hours of incubation a survival proportional to the molarity of the solution with a IC50, the concentration at which $50 \%$ of the cells do not survive, equal to $0.00009 \mu \mathrm{M}$ for dasatinib, $0.0065 \mu \mathrm{M}$ for ponatinib and $4 \mu \mathrm{M}$ for pimozide (Figure 2).

Combination exposition revealed a synergic effect of the drugs tested since the combination index $(\mathrm{Cl})$ according to Webb's fractional product revealed a value of 0.66 and 0.8 respectively when exposing the $\mathrm{K} 562$ cells to dasatinib $0.00005 \mu \mathrm{M}$ plus pimozide $2.5 \mu \mathrm{M}$, and dasatinib $0.00001 \mu \mathrm{M}$ plus pimozide $2.5 \mu \mathrm{M}$ (Figure 3).

Sinergy was demonstrated to be present in the association ponatinibpimozide, too, since $\mathrm{Cl}$ was 0.46 and 0.61 when $\mathrm{K} 562$ cells were exposed to ponatinib $0.005 \mu \mathrm{M}$ plus pimozide $2.5 \mu \mathrm{M}$ and ponatinib $0.0025 \mu \mathrm{M}$, respectively. 


\section{CHAPTER}

\section{Discussion}

$\mathrm{CML}$ is a neoplasm of the myeloid-committed hematopoietic stem cell characterized by the translocation between the chromosome 9 and 22, that determines the formation of a chimeric gene, BCRABL, named after the two genes that are involved in the chromosomal aberration.

In the last decades, the use of TKIs has dramatically improved CML patients' survival, as it is today similar to the one of the general population. Nonetheless, a few patients experience resistance to tyrosine kinase inhibition due to either additional point mutations occurring on the BCRABL gene that modify the molecular affinity of the TKI to the chimeric protein or other several escape pathways activation that are only in part known this far.

The STAT family proteins, and in particular STAT3 and STAT5A and $B$ are involved in the transduction of survival and proliferative signals coming from various upstream molecules, among which also ABL. Thus, STAT5 has already been identified as a potential target of therapy in CML models $(55,74,75)$. 
A molecule of common use in clinical practice as a neuroleptic drug, pimozide, has been found to exert a significant inhibition of STAT5 in 2011 by Nelson et al (76). Since then, other authors studied with success the possible application of pimozide to the direct inhibition of the growth of cell lines of CML. Moreover, derivatives of pimozide, obtained removing, modifying or adding chemical groups to these molecules in the effort of design a more potent and less toxic compound, were synthetized and tested on CML cell-lines to demonstrate efficacy in cell growth inhibition as single agent and in association with imatinib (74).

In the present experiment, we aimed at demonstrating how pimozide may determine inhibition of $\mathrm{K} 562$ cell growth in association with second- and third-generation TKIs.

The Webb's formula for drug interaction demonstrated that pimozide at a low concentration has a synergic effect with dasatinib and/or ponatinib, at doses that are significantly lower than the IC50 of the single agent tested on K562 cells.

In particular, the association dasatinib-pimozide at a molarity of 0.00005- $2.5 \mu \mathrm{M}$ determined a survival of $45 \%$ of the control with a Webb's $\mathrm{Cl}$ of 0.66 , while the combination ponatinib-pimozide at a concentration of $0.005-2.5 \mu \mathrm{M}$ was associated with $40 \%$ survival, with a $\mathrm{Cl}$ of 0.46 .

Both these data are of note, resembling that almost half of the concentration of TKI used to obtain around $50 \%$ inhibition of cell growth in a culture of K562 cells determine the same effect as the full concentration, if associated with an equally reduced concentration of 
pimozide. Since STAT5 was recognized as a key molecule in a lot of hematologic malignancies, pimozide and/or its derivatives may represent a potential, new target-therapy in the armamentarium in the treatment of CML. If pimozide would express the same inhibiting activity in an in vivo experiment, this evidence may open the door to a possible therapeutic association with TKIs with evident benefits versus the current treatment based on single-target BCRABL1 inhibition.

New perspectives of this ongoing study comprise the extension of the work on the leukemic stem cells. Persistence of vital, quiescent LSC in CML and their capability of escape the TKIs mechanism of action is still a major issue in clinical practice. In fact, it is still not clear why patients with the same BCRABL transcript, age, history of the disease, kind and timing of deep molecular response, may have a very different treatment-free-remission duration. The application of a new drug for CML, with the possibility of "multi-targeting" the disease, is surely of great interest and may solve or attenuate the problem of TKI-resistant patients or the one of the increasing toxicity that often occurs in patients with at least one TKI switch.

The present study is of key importance in opening new fields of research that may lead to the development of $\mathrm{CML}$ treatment schemes considering a multi-target approach that should overcome or prevent resistance and, at the same time, may sensibly lower the rate of TKI-related toxicity that nowadays represent a major issue and the cost-to-pay for all the patients assuming these drugs in order to achieve the desired survival expectations. 


\section{Tables and figures}

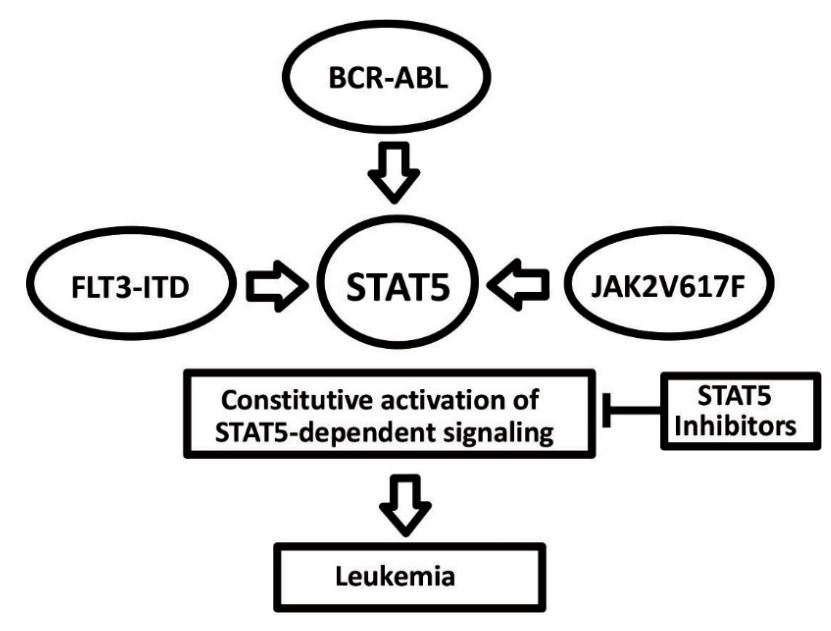

Figure 1. BCRABL chimeric transcript, FLT3 mutations and the JAK2 V617F mutation all converging into STAT5.

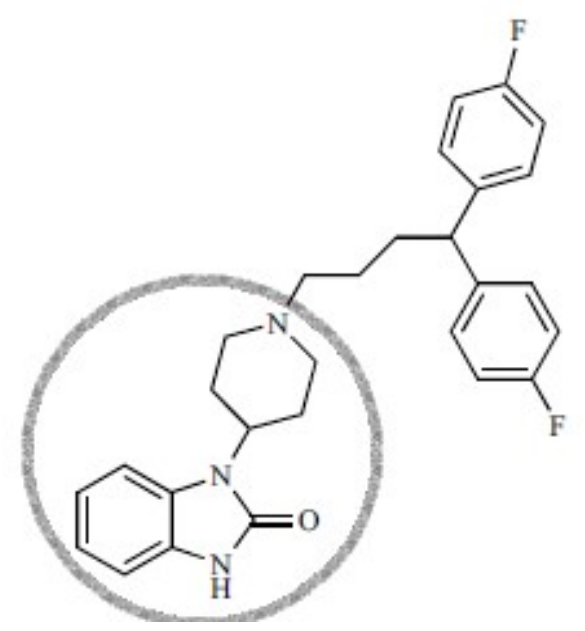

Figure 2. Chemical structure of Pimozide. The circle indicates the functional benzimidazolinone-piperidine group. 


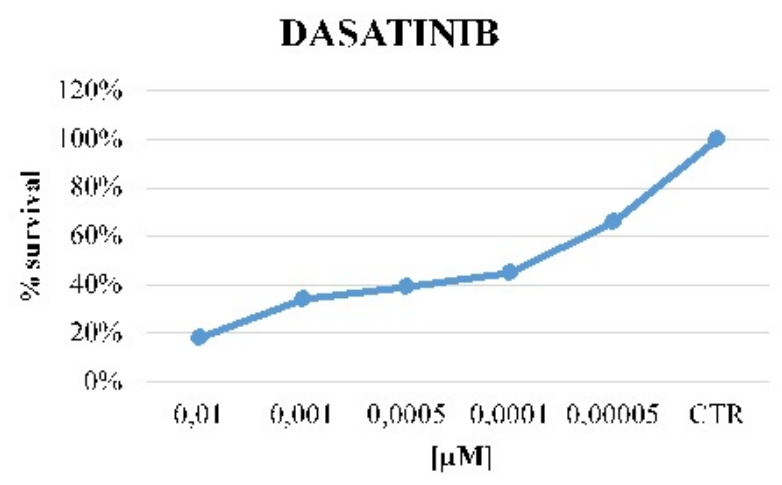

\begin{tabular}{|c|c|}
\hline $\begin{array}{c}\text { Concentration } \\
\text { [pM] }\end{array}$ & $\begin{array}{c}\text { Survival } \\
\%\end{array}$ \\
\hline 0,01 & $18 \%$ \\
\hline 0,001 & $34 \%$ \\
\hline 0,0005 & $39 \%$ \\
\hline 0,0001 & $45 \%$ \\
\hline 0,00005 & $66 \%$ \\
\hline CTR & $100 \%$ \\
\hline
\end{tabular}
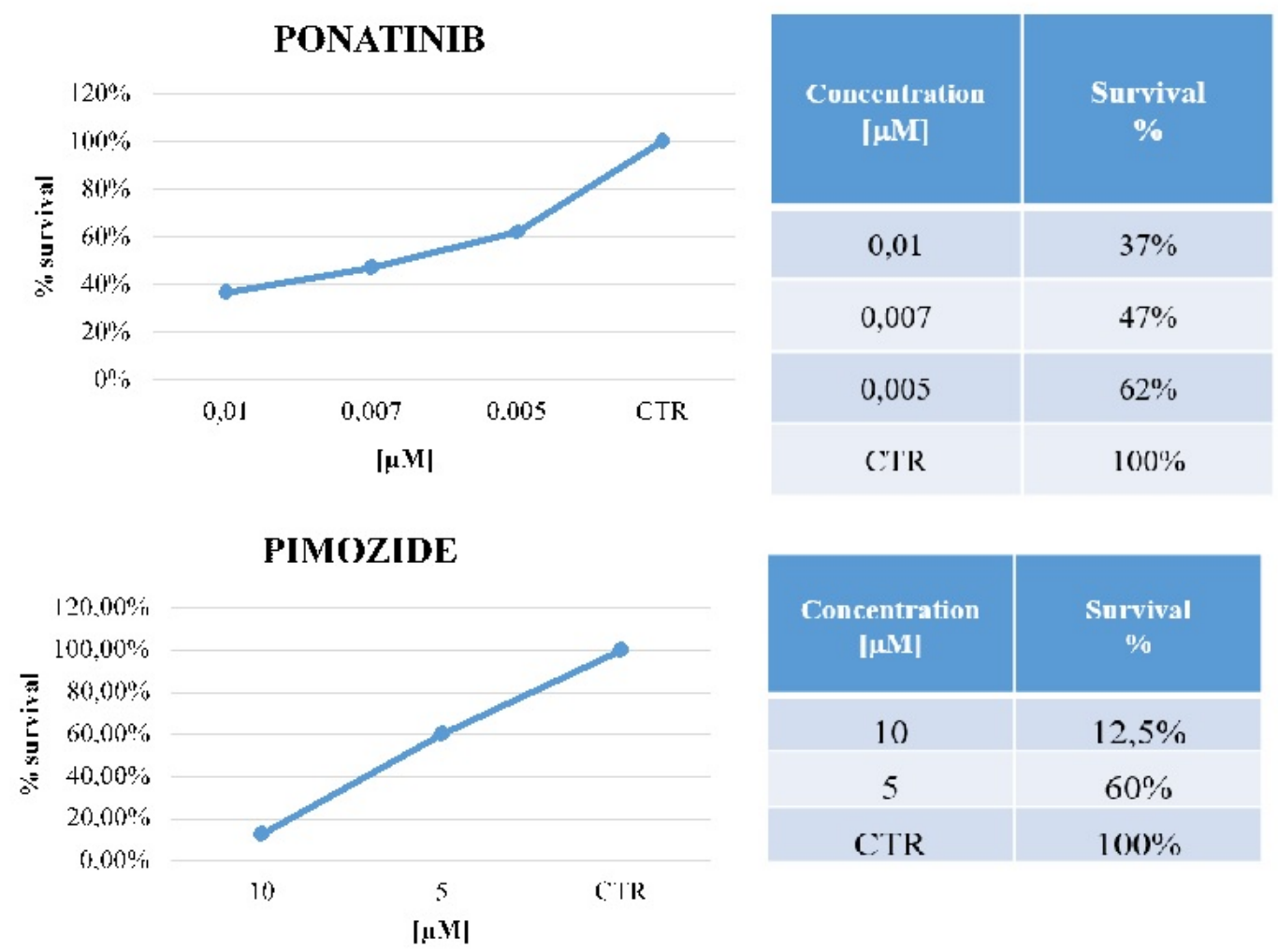

Figure 3. Percentage of surviving cells of K562 lines exposed to increasing single drug concentrations (expressed in $\mu \mathrm{M}$ ) compared to a control culture without any inhibitor. IC50 dasatinib $0.00009 \mu \mathrm{M}$, ponatinib 0.0065, Pimozide $4 \mu \mathrm{M}$. 


\begin{tabular}{|c|c|c|c|}
\hline $\begin{array}{c}\text { Concentration } \\
{[\mu \mathrm{M}]}\end{array}$ & $\begin{array}{c}\text { Survival } \\
\% \%\end{array}$ & $\begin{array}{c}\text { Concentration } \\
{[\mu \mathrm{M}]}\end{array}$ & $\begin{array}{c}\text { Survival } \\
\%\end{array}$ \\
\hline CTR & $100 \%$ & \multirow{2}{*}{ CTR } & \multirow{2}{*}{$100 \%$} \\
\hline \multirow{2}{*}{ Pimozide 2,5} & \multirow{2}{*}{$94 \%$} & & \\
\hline & & Pimozide 2,5 & $94 \%$ \\
\hline Dasatinb 0,00005 & $72 \%$ & Ponatinib 0,005 & $90 \%$ \\
\hline $\begin{array}{c}\text { Dasatinb } 0,00005 \\
+ \text { Pimozide } 2,5\end{array}$ & $45 \%$ & $\begin{array}{l}\text { Ponatinib } 0,005 \\
+ \text { Pimozide } 2,5\end{array}$ & $39 \%$ \\
\hline Dasatinib 0,00001 & $77 \%$ & Ponatinib 0.0025 & $100 \%$ \\
\hline $\begin{array}{c}\text { Dasatinib } 0,00001 \\
+ \text { Pimozide } 2,5\end{array}$ & $58 \%$ & $\begin{array}{c}\text { Ponatinib } 0,0025 \\
\text { + Pimozide } 2,5\end{array}$ & $58 \%$ \\
\hline CI 1 & 0,66 & CI 1 & 0.46 \\
\hline CI 2 & $\mathbf{0 , 8 0}$ & CI 2 & 0.61 \\
\hline
\end{tabular}

Figure 4. Study of the association effect. $\mathrm{Cl}$ (Combination Index) for both Dasatinib + Pimozide and Ponatinib + Pimozide reveals synergism in determining inhibition of cell growth in K562 cell lines. 


\section{Bibliography}

1. Nadal E, Olavarria E. Imatinib mesylate (Gleevec/Glivec) a molecular-targeted therapy for chronic myeloid leukaemia and other malignancies. Int J Clin Pract. 2004 May;58(5):511-6.

2. Cuellar S, Vozniak M, Rhodes J, Forcello N, Olszta D. BCR-ABL1 tyrosine kinase inhibitors for the treatment of chronic myeloid leukemia. J Oncol Pharm Pract. 2018 Sep;24(6):433-52.

3. Molica M, Naqvi K, Cortes JE, Paul S, Kadia TM, Breccia M, et al. Treatment-free remission in chronic myeloid leukemia. Clin Adv Hematol Oncol. 2019 Dec;17(12):686-96.

4. Baccarani M, Abruzzese E, Accurso V, Albano F, Annunziata M, Barulli $S$, et al. Managing chronic myeloid leukemia for treatment-free remission: a proposal from the GIMEMA CML WP. Blood Adv. 2019 23;3(24):4280-90.

5. Rea D, Cayuela J-M. Treatment-free remission in patients with chronic myeloid leukemia. Int J Hematol. 2018 Oct;108(4):355-64.

6. Bower H, Björkholm M, Dickman PW, Höglund M, Lambert PC, Andersson TM-L. Life Expectancy of Patients With Chronic Myeloid Leukemia Approaches the Life Expectancy of the General Population. J Clin Oncol. 2016 20;34(24):2851-7.

7. Pfirrmann M, Baccarani M, Saussele S, Guilhot J, Cervantes F, Ossenkoppele G, et al. Prognosis of long-term survival considering disease-specific death in patients with chronic myeloid leukemia. Leukemia. 2016 Jan;30(1):48-56.

8. Sasaki K, Kantarjian HM, Jain P, Jabbour EJ, Ravandi F, Konopleva $\mathrm{M}$, et al. Conditional survival in patients with chronic myeloid leukemia in chronic phase in the era of tyrosine kinase inhibitors. Cancer. 2016 Jan 15;122(2):238-48.

9. Chomel JC, Bonnet ML, Sorel N, Sloma I, Bennaceur-Griscelli A, Rea $D$, et al. Leukemic stem cell persistence in chronic myeloid leukemia patients in deep molecular response induced by tyrosine 
kinase inhibitors and the impact of therapy discontinuation. Oncotarget. 2016 May 5;7(23):35293-301.

10. Chomel J-C, Bonnet M-L, Sorel N, Bertrand A, Meunier M-C, Fichelson $\mathrm{S}$, et al. Leukemic stem cell persistence in chronic myeloid leukemia patients with sustained undetectable molecular residual disease. Blood. 2011 Sep 29;118(13):3657-60.

11. Bhatia R, Holtz M, Niu N, Gray R, Snyder DS, Sawyers CL, et al. Persistence of malignant hematopoietic progenitors in chronic myelogenous leukemia patients in complete cytogenetic remission following imatinib mesylate treatment. Blood. 2003 Jun 15;101(12):4701-7.

12. Chu S, McDonald T, Lin A, Chakraborty S, Huang Q, Snyder DS, et al. Persistence of leukemia stem cells in chronic myelogenous leukemia patients in prolonged remission with imatinib treatment. Blood. 2011 Nov 17;118(20):5565-72.

13. Holyoake TL, Vetrie D. The chronic myeloid leukemia stem cell: stemming the tide of persistence. Blood. 2017 23;129(12):1595-606.

14. Chronic Myeloid Leukemia - Cancer Stat Facts [Internet]. SEER. [cited 2021 Jun 4]. Available from: https://seer.cancer.gov/statfacts/html/cmyl.html15. Thielen N, Visser O, Ossenkoppele G, Janssen J. Chronic myeloid leukemia in the Netherlands: a population-based study on incidence, treatment, and survival in 3585 patients from 1989 to 2012. European Journal of Haematology. 2016;97(2):145-54.

16. Sasaki K, Strom SS, O'Brien S, Jabbour E, Ravandi F, Konopleva $M$, et al. Relative survival in patients with chronic-phase chronic myeloid leukaemia in the tyrosine-kinase inhibitor era: analysis of patient data from six prospective clinical trials. The Lancet Haematology. 2015 May 1;2(5):e186-93.

17. Arber DA, Orazi A, Hasserjian R, Thiele J, Borowitz MJ, Le Beau $\mathrm{MM}$, et al. The 2016 revision to the World Health Organization classification of myeloid neoplasms and acute leukemia. Blood. 2016 19;127(20):2391-405.

18. Hochhaus A, Baccarani M, Silver RT, Schiffer C, Apperley JF, Cervantes F, et al. European LeukemiaNet 2020 recommendations for treating chronic myeloid leukemia. Leukemia. 2020 Apr;34(4):96684.

19. Roskoski R. Classification of small molecule protein kinase inhibitors based upon the structures of their drug-enzyme complexes. Pharmacol Res. 2016 Jan;103:26-48.

20. Rossari F, Minutolo F, Orciuolo E. Past, present, and future of Bcr-Abl inhibitors: from chemical development to clinical efficacy. J 
Hematol Oncol [Internet]. 2018 Jun 20 [cited 2021 Jun 7];11. Available from: https://www.ncbi.nlm.nih.gov/pmc/articles/PMC6011351/

21. Jabbour $\mathrm{E}$, Kantarjian $\mathrm{H}$, Cortes J. Use of second- and thirdgeneration tyrosine kinase inhibitors in the treatment of chronic myeloid leukemia: an evolving treatment paradigm. Clin Lymphoma Myeloma Leuk. 2015 Jun;15(6):323-34.

22. Vener C, Banzi R, Ambrogi F, Ferrero A, Saglio G, Pravettoni G, et al. First-line imatinib vs second- and third-generation TKIs for chronic-phase CML: a systematic review and meta-analysis. Blood Adv. 2020 Jun 23;4(12):2723-35.

23. Campiotti L, Suter MB, Guasti L, Piazza R, Gambacorti-Passerini C, Grandi $A M$, et al. Imatinib discontinuation in chronic myeloid leukaemia patients with undetectable BCR-ABL transcript level: A systematic review and a meta-analysis. European Journal of Cancer. 2017 May 1;77:48-56.

24. Fava C, Rege-Cambrin G, Dogliotti I, Cerrano M, Berchialla P, Dragani $M$, et al. Observational study of chronic myeloid leukemia Italian patients who discontinued tyrosine kinase inhibitors in clinical practice. 1. 2019 Aug 1;104(8):1589-96.

25. Saussele S, Richter J, Guilhot J, Gruber FX, Hjorth-Hansen H, Almeida $A$, et al. Discontinuation of tyrosine kinase inhibitor therapy in chronic myeloid leukaemia (EURO-SKI): a prespecified interim analysis of a prospective, multicentre, non-randomised, trial. The Lancet Oncology. 2018 Jun 1;19(6):747-57.

26. Rea D, Nicolini FE, Tulliez M, Guilhot F, Guilhot J, Guerci-Bresler $A$, et al. Discontinuation of dasatinib or nilotinib in chronic myeloid leukemia: interim analysis of the STOP 2G-TKI study. Blood. 2017 Feb 16;129(7):846-54.

27. Dragani M, Rege Cambrin G, Berchialla P, Dogliotti I, Rosti G, Castagnetti $F$, et al. A Retrospective Analysis about Frequency of Monitoring in Italian Chronic Myeloid Leukemia Patients after Discontinuation. J Clin Med. 2020 Nov 17;9(11).

28. Hughes TP, Mauro MJ, Cortes JE, Minami H, Rea D, DeAngelo DJ, et al. Asciminib in Chronic Myeloid Leukemia after ABL Kinase Inhibitor Failure. New England Journal of Medicine. 2019 Dec 12;381(24):2315-26.

29. Winer ES, DeAngelo DJ. A Review of Omacetaxine: A Chronic Myeloid Leukemia Treatment Resurrected. Oncol Ther. 2018 Jun $1 ; 6(1): 9-20$.

30. Hu B, Lin X, Lee HC, Huang X, Tidwell RSS, Ahn KW, et al. Timing of allogeneic hematopoietic cell transplantation (alloHCT) for chronic myeloid leukemia (CML) patients. Leuk Lymphoma. 2020 
31. Corbin AS, Agarwal A, Loriaux M, Cortes J, Deininger MW, Druker BJ. Human chronic myeloid leukemia stem cells are insensitive to imatinib despite inhibition of BCR-ABL activity. J Clin Invest. 2011 Jan;121(1):396-409.

32. Hamilton A, Helgason GV, Schemionek M, Zhang B, Myssina S, Allan EK, et al. Chronic myeloid leukemia stem cells are not dependent on Bcr-Abl kinase activity for their survival. Blood. 2012 Feb 9;119(6):1501-10.

33. Yuda J, Miyamoto T, Odawara J, Ohkawa Y, Semba Y, Hayashi $M$, et al. Persistent detection of alternatively spliced BCR-ABL variant results in a failure to achieve deep molecular response. Cancer Sci. 2017 Nov;108(11):2204-12.

34. Kinstrie R, Horne GA, Morrison H, Irvine D, Munje C, Castañeda $E G$, et al. CD93 is expressed on chronic myeloid leukemia stem cells and identifies a quiescent population which persists after tyrosine kinase inhibitor therapy. Leukemia. 2020;34(6):1613-25.

35. Jeanpierre S, Arizkane K, Thongjuea S, Grockowiak E, Geistlich $\mathrm{K}$, Barral $\mathrm{L}$, et al. The quiescent fraction of chronic myeloid leukemic stem cells depends on BMPR1B, Stat3 and BMP4-niche signals to persist in patients in remission. Haematologica. 2020 Jan 30;

36. Silvestri G, Trotta R, Stramucci L, Ellis JJ, Harb JG, Neviani P, et al. Persistence of Drug-Resistant Leukemic Stem Cells and Impaired NK Cell Immunity in CML Patients Depend on MIR300 Antiproliferative and PP2A-Activating Functions. Blood Cancer Discov. 2020 Jul 1;1(1):48-67.

37. Pellicano F, Scott MT, Helgason GV, Hopcroft LEM, Allan EK, Aspinall-O'Dea $M$, et al. The antiproliferative activity of kinase inhibitors in chronic myeloid leukemia cells is mediated by FOXO transcription factors. Stem Cells. 2014 Sep;32(9):2324-37.

38. Hurtz C, Hatzi K, Cerchietti L, Braig M, Park E, Kim Y, et al. BCL6-mediated repression of p53 is critical for leukemia stem cell survival in chronic myeloid leukemia. J Exp Med. 2011 Oct 24;208(11):2163-74.

39. Pellicano F, Holyoake TL. Assembling defenses against therapyresistant leukemic stem cells: Bcl6 joins the ranks. J Exp Med. 2011 Oct 24;208(11):2155-8.

40. Naka K, Hoshii T, Muraguchi T, Tadokoro Y, Ooshio T, Kondo Y, et al. TGF-beta-FOXO signalling maintains leukaemia-initiating cells in chronic myeloid leukaemia. Nature. 2010 Feb 4;463(7281):676-80.

41. Zhao C, Chen A, Jamieson $\mathrm{CH}$, Fereshteh $\mathrm{M}$, Abrahamsson $\mathrm{A}$, Blum $J$, et al. Hedgehog signalling is essential for maintenance of 
cancer stem cells in myeloid leukaemia. Nature. 2009 Apr 9;458(7239):776-9.

42. Dierks C, Beigi R, Guo G-R, Zirlik K, Stegert MR, Manley P, et al. Expansion of Bcr-Abl-positive leukemic stem cells is dependent on Hedgehog pathway activation. Cancer Cell. 2008 Sep 9;14(3):238-49.

43. Irvine DA, Zhang B, Kinstrie R, Tarafdar A, Morrison H, Campbell $\mathrm{VL}$, et al. Deregulated hedgehog pathway signaling is inhibited by the smoothened antagonist LDE225 (Sonidegib) in chronic phase chronic myeloid leukaemia. Scientific Reports. 2016 May 9;6(1):25476.

44. Lim CP, Cao X. Structure, function, and regulation of STAT proteins. Mol Biosyst. 2006 Nov;2(11):536-50.

45. Jatiani SS, Baker SJ, Silverman LR, Reddy EP. Jak/STAT pathways in cytokine signaling and myeloproliferative disorders: approaches for targeted therapies. Genes Cancer. 2010 Oct;1(10):979-93.

46. de Groot RP, Raaijmakers JA, Lammers JW, Koenderman L. STAT5-Dependent CyclinD1 and Bcl-xL expression in Bcr-Abltransformed cells. Mol Cell Biol Res Commun. 2000 May;3(5):299305.

47. Magné S, Caron S, Charon M, Rouyez M-C, Dusanter-Fourt I. STAT5 and Oct-1 Form a Stable Complex That Modulates Cyclin D1 Expression. Mol Cell Biol. 2003 Dec;23(24):8934-45.

48. STAT5 signaling is required for the efficient induction and maintenance of CML in mice - PubMed [Internet]. [cited 2021 Jun 11]. Available from: https://pubmed.ncbi.nlm.nih.gov/16522816/

49. Warsch W, Grundschober E, Sexl V. Adding a new facet to STAT5 in CML: multitasking for leukemic cells. Cell Cycle. 2013 Jun $15 ; 12(12): 1813-4$.

50. Papaemmanuil E, Gerstung M, Bullinger L, Gaidzik VI, Paschka $P$, Roberts ND, et al. Genomic Classification and Prognosis in Acute Myeloid Leukemia. N Engl J Med. 2016 Jun 9;374(23):2209-21.

51. Fischer $M$, Schnetzke $U$, Spies-Weisshart $B$, Walther $M$, Fleischmann M, Hilgendorf I, et al. Impact of FLT3-ITD diversity on response to induction chemotherapy in patients with acute myeloid leukemia. Haematologica. 2017 Apr;102(4):e129-31.

52. Gilliland DG, Griffin JD. The roles of FLT3 in hematopoiesis and leukemia. Blood. 2002 Sep 1;100(5):1532-42.

53. Baxter EJ, Scott LM, Campbell PJ, East C, Fourouclas N, Swanton S, et al. Acquired mutation of the tyrosine kinase JAK2 in human myeloproliferative disorders. Lancet. 2005 Mar 19;365(9464):1054-61. 
54. Yan D, Hutchison RE, Mohi G. Critical requirement for Stat5 in a mouse model of polycythemia vera. Blood. $2012 \mathrm{Apr}$ 12;119(15):3539-49.

55. Tolomeo M, Meli M, Grimaudo S. STAT5 and STAT5 Inhibitors in Hematological Malignancies. Anticancer Agents Med Chem. 2019;19(17):2036-46.

56. Lee T-S, Ma W, Zhang X, Giles F, Cortes J, Kantarjian H, et al. BCR-ABL alternative splicing as a common mechanism for imatinib resistance: evidence from molecular dynamics simulations. Mol Cancer Ther. 2008 Dec;7(12):3834-41.

57. Ma W, Kantarjian H, Yeh C-H, Zhang ZJ, Cortes J, Albitar M. BCR-ABL Truncation due to Premature Translation Termination as a Mechanism of Resistance to Kinase Inhibitors. Acta Haematol. 2009 Apr;121(1):27-31.

58. White DL, Saunders VA, Dang P, Engler J, Zannettino ACW, Cambareri AC, et al. OCT-1-mediated influx is a key determinant of the intracellular uptake of imatinib but not nilotinib (AMN107): reduced OCT-1 activity is the cause of low in vitro sensitivity to imatinib. Blood. 2006 Jul 15;108(2):697-704.

59. White DL, Dang P, Engler J, Frede A, Zrim S, Osborn M, et al. Functional activity of the OCT-1 protein is predictive of long-term outcome in patients with chronic-phase chronic myeloid leukemia treated with imatinib. J Clin Oncol. 2010 Jun 1;28(16):2761-7.

60. Shah NP, Nicoll JM, Nagar B, Gorre ME, Paquette RL, Kuriyan J, et al. Multiple BCR-ABL kinase domain mutations confer polyclonal resistance to the tyrosine kinase inhibitor imatinib (STI571) in chronic phase and blast crisis chronic myeloid leukemia. Cancer Cell. 2002 Aug;2(2):117-25.

61. Branford S, Rudzki Z, Walsh S, Parkinson I, Grigg A, Szer J, et al. Detection of BCR-ABL mutations in patients with CML treated with imatinib is virtually always accompanied by clinical resistance, and mutations in the ATP phosphate-binding loop (P-loop) are associated with a poor prognosis. Blood. 2003 Jul 1;102(1):276-83.

62. Hochhaus A, Kreil S, Corbin AS, La Rosée P, Müller MC, Lahaye $\mathrm{T}$, et al. Molecular and chromosomal mechanisms of resistance to imatinib (STI571) therapy. Leukemia. 2002 Nov;16(11):2190-6.

63. von Bubnoff N, Peschel C, Duyster J. Resistance of Philadelphiachromosome positive leukemia towards the kinase inhibitor imatinib (STI571, Glivec): a targeted oncoprotein strikes back. Leukemia. 2003 May;17(5):829-38.

64. Willis SG, Lange $T$, Demehri $S$, Otto $S$, Crossman L, Niederwieser $D$, et al. High-sensitivity detection of BCR-ABL kinase 
domain mutations in imatinib-naive patients: correlation with clonal cytogenetic evolution but not response to therapy. Blood. 2005 Sep 15;106(6):2128-37.

65. Hochhaus A, La Rosée P, Müller MC, Ernst T, Cross NCP. Impact of BCR-ABL mutations on patients with chronic myeloid leukemia. Cell Cycle. 2011 Jan 15;10(2):250-60.

66. Liu J, Yang H, Xu X, Yi S, Meng L. Mutations in the BCR-ABL1 kinase domain in patients with chronic myeloid leukaemia treated with TKIs or at diagnosis. Oncol Lett. 2020 Aug;20(2):1071-6.

67. Vainchenker W, Constantinescu SN. JAK/STAT signaling in hematological malignancies. Oncogene. 2013 May 23;32(21):260113.

68. Berger A, Sexl V, Valent P, Moriggl R. Inhibition of STAT5: a therapeutic option in BCR-ABL1-driven leukemia. Oncotarget. 2014 Oct 30;5(20):9564-76.

69. Warsch W, Grundschober E, Berger A, Gille L, Cerny-Reiterer S, Tigan A-S, et al. STAT5 triggers BCR-ABL1 mutation by mediating ROS production in chronic myeloid leukaemia. Oncotarget. 2012 Dec;3(12):1669-87.

70. Warsch W, Walz C, SexI V. JAK of all trades: JAK2-STAT5 as novel therapeutic targets in BCR-ABL1+ chronic myeloid leukemia. Blood. 2013 Sep 26;122(13):2167-75.

71. Shuai K, Halpern J, ten Hoeve J, Rao X, Sawyers CL. Constitutive activation of STAT5 by the BCR-ABL oncogene in chronic myelogenous leukemia. Oncogene. 1996 Jul 18;13(2):247-54.

72. Nieborowska-Skorska M, Wasik MA, Slupianek A, Salomoni $P$, Kitamura T, Calabretta B, et al. Signal transducer and activator of transcription (STAT) 5 activation by $B C R / A B L$ is dependent on intact Src homology $(\mathrm{SH}) 3$ and $\mathrm{SH} 2$ domains of $B C R / A B L$ and is required for leukemogenesis. J Exp Med. 1999 Apr 19;189(8):1229-42.

73. Kosova B, Tezcanli B, Ekiz HA, Cakir Z, Selvi N, Dalmizrak A, et al. Suppression of STAT5A increases chemotherapeutic sensitivity in imatinib-resistant and imatinib-sensitive K562 cells. Leuk Lymphoma. 2010 Oct;51(10):1895-901.

74. Rondanin R, Simoni D, Romagnoli R, Baruchello R, Marchetti P, Costantini $\mathrm{C}$, et al. Inhibition of activated STAT5 in Bcr/Abl expressing leukemia cells with new pimozide derivatives. Bioorg Med Chem Lett. 2014 Sep 15;24(18):4568-74.

75. Rondanin R, Simoni D, Maccesi M, Romagnoli R, Grimaudo S, Pipitone RM, et al. Effects of Pimozide Derivatives on pSTAT5 in K562 Cells. ChemMedChem. 2017 Aug 8;12(15):1183-90. 
76. Nelson EA, Walker SR, Weisberg E, Bar-Natan M, Barrett R, Gashin LB, et al. The STAT5 inhibitor pimozide decreases survival of chronic myelogenous leukemia cells resistant to kinase inhibitors. Blood. 2011 Mar 24;117(12):3421-9. 